\title{
Role of Ultrasonography Otolaryngology
}

\author{
Rezaul Karim ${ }^{1}$
}

\section{ABSTRACT}

Ultrasonography (US) is frequently requested by the otolaryngologists in their day to day practice. Though US assessment is sensitive and in many situations, specific investigation for prognosis and management of patients, FNAC and Ultrasonography carries more predictive value. Ultrasonography is very useful for assessment of neck nodes and in combination with CT scan is an excellent imaging tool for follow up of head and neck cancers. Inflammatory neck nodes vary in characteristics from neoplastic nodes and US can classify them with fair degree of predictability. Thyroid nodules should strictly follow standard protocol of management, as most of the masses are benign and unnecessary diagnostic or therapeutic interventions are not required. Kim's criteria and American Association for Clinical Endocrinology recommendations are sensitive and specific for offering systematic guidance for management of thyroid nodules. US have limited roles in the assessments of sialadenitis, Sialolithiasis and salivary tumors. US is an effective tool in guiding biopsies and aspirations for diagnostic and therapeutic purposes.

Kevwords

Ultrasonography; Diagnosis; Lymph Nodes; Thyroid Nodule; Salivary Glands

$\mathrm{U}$ ltrasonography is a low cost, easily available tool. While Conventional Radiography, MDCT and MRI are the preferred diagnostic armamentarium for the otolaryngologist, role of Ultrasonography in the assessment of neck cannot be overemphasized. Ultrasonography has the traditional role in distinguishing solid and cystic masses but with the advent of more and more sophisticated ultrasound machines, the sensitivity, specificity and predictive values of detected lesions have substantially improved. I present here a brief review of the usefulness of Ultrasonography as a diagnostic tool useful for the otolaryngologist.

\section{Assessment of Neck Nodes}

Cervical lymphadenopathy is a common problem affecting all age groups. The sonographic appearance of normal nodes is different from abnormal nodes. Gray

1 - Department of Radiology, College of Medicine and Sagar Dutta Hospital, Kolkata

\section{Corresponding author:}

Dr Rezaul Karim

email: karim_rex@yahoo.co.in scale ultrasound assessment is used for evaluation of the number, size, shape, margin, hilum, matting and perilymph-node fluid collection, oedema, caseous necrosis and abscess formation while colour and power Doppler are utilised for evaluation of the intranodal vascularity. ${ }^{1}$

In sonography neck nodes are classified into eight zones- submental, submandibular, parotid, upper cervical, middle cervical, lower cervical, supraclavicular and posterior triangle (Fig.1). ${ }^{1}$ Routine sonographic assessment shows small normal lymph nodes in the cervical region. These nodes are oval in shape (i.e., wider than their length) having echogenic hilum. The cortex and medulla of lymph nodes are easily identified. When the nodes are larger $(>9 \mathrm{~mm})$, round, hypoechoic and vascular, these are more likely to be pathological. Reactive and cancerous lymph nodes can be differentiated by an experienced sonographer. The reactive nodes (Fig.2) are usually oval (the long axis short axis ratio is around 0.5) matted or non-matted have cortico-medullary differentiation and shows ample vascularity with low pulsatility and resistance whereas the malignant nodes are round, hypoechoic, show no cortico-medullary differentiation and have multiple 
vessels in the parenchyma. Lymph nodes loaded with malignant cells are hypoechoic in US and the echogenic hilum is not seen. Solbaiti ${ }^{2}$ showed that only $4 \%$ of malignant nodes show echogenic hilum. Most agree that benign nodes show hilar echogenicity though a few investigators (Vassalo et $\mathrm{al}^{3}$ ) published just the contrary findings. However, no single criterion is foolproof and with experience, investigators learn to distinguish different categories of lymph nodes fairly accurately.

Colour and power Doppler sonography predict the vascularity of the node fairly accurately. Power Doppler is more sensitive in detection of flow in low-flow in small vessels. For effective power Doppler assessment standardized setting must be used to detect low-velocity flow. ${ }^{4}$ Ariji et al ${ }^{5}$ described a unique technique of power Doppler assessment of metastatic lymph node in head and neck cancer. First, CT scan of neck is performed to detect the lymph nodes, followed by assessment by power Doppler sonography. The nodes with transverse length/longitudinal length of 0.65 which have no detectable hilum fulfilling power Doppler characteristics are considered metastatic. The colour Doppler signals from lymph nodes are of three types: hilar, parenchymal (Fig. 3) and no signal. Metastatic lymph nodes are more likely to show parenchymal signal whereas reactive nodes show hilar signal. By this technique, the

Table I : Algorithm followed by Ariji et al $^{5}$ for radiological diagnosis of enlarged lymph nodes

Lymphnode seen on enhanced CT
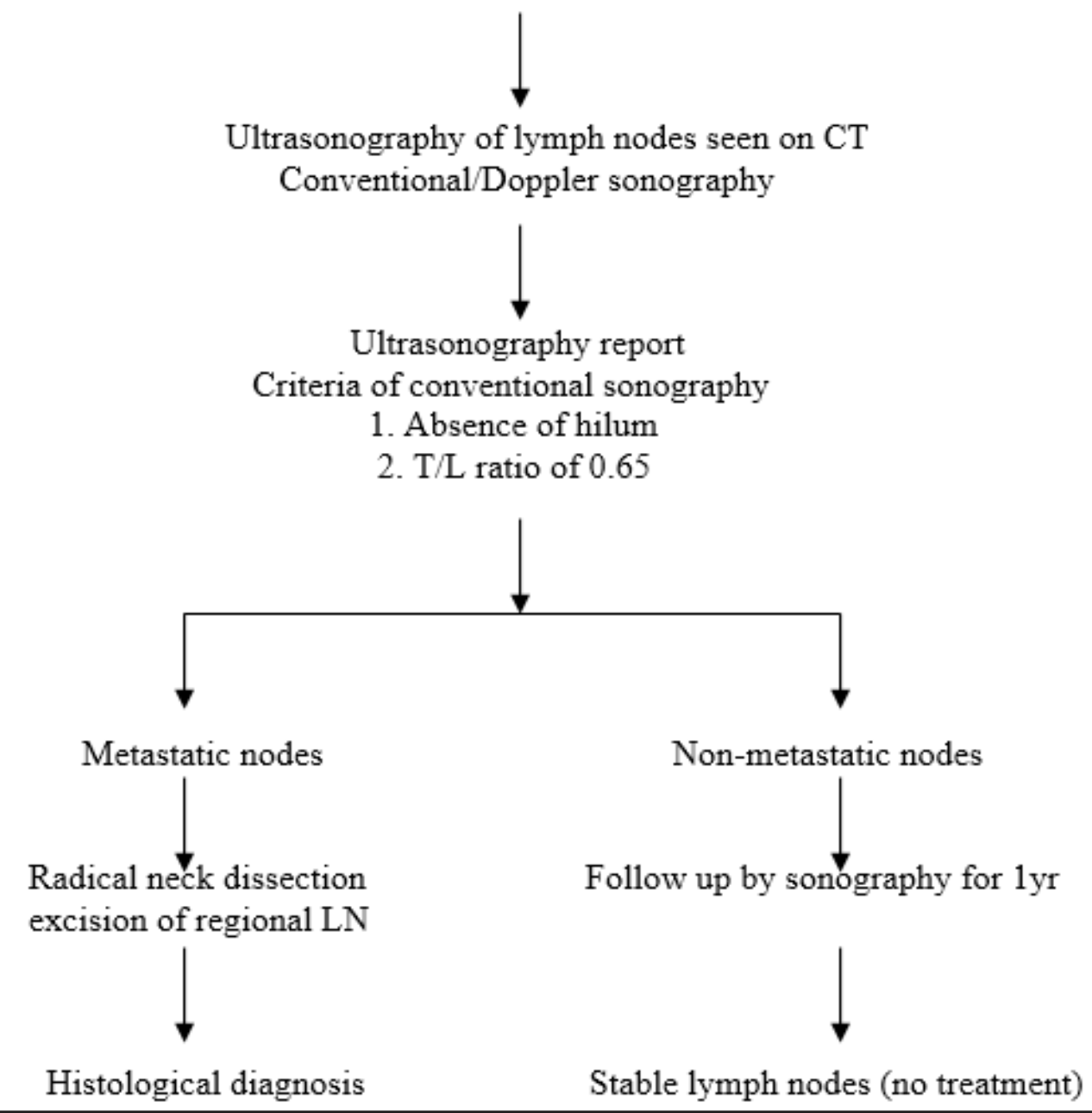


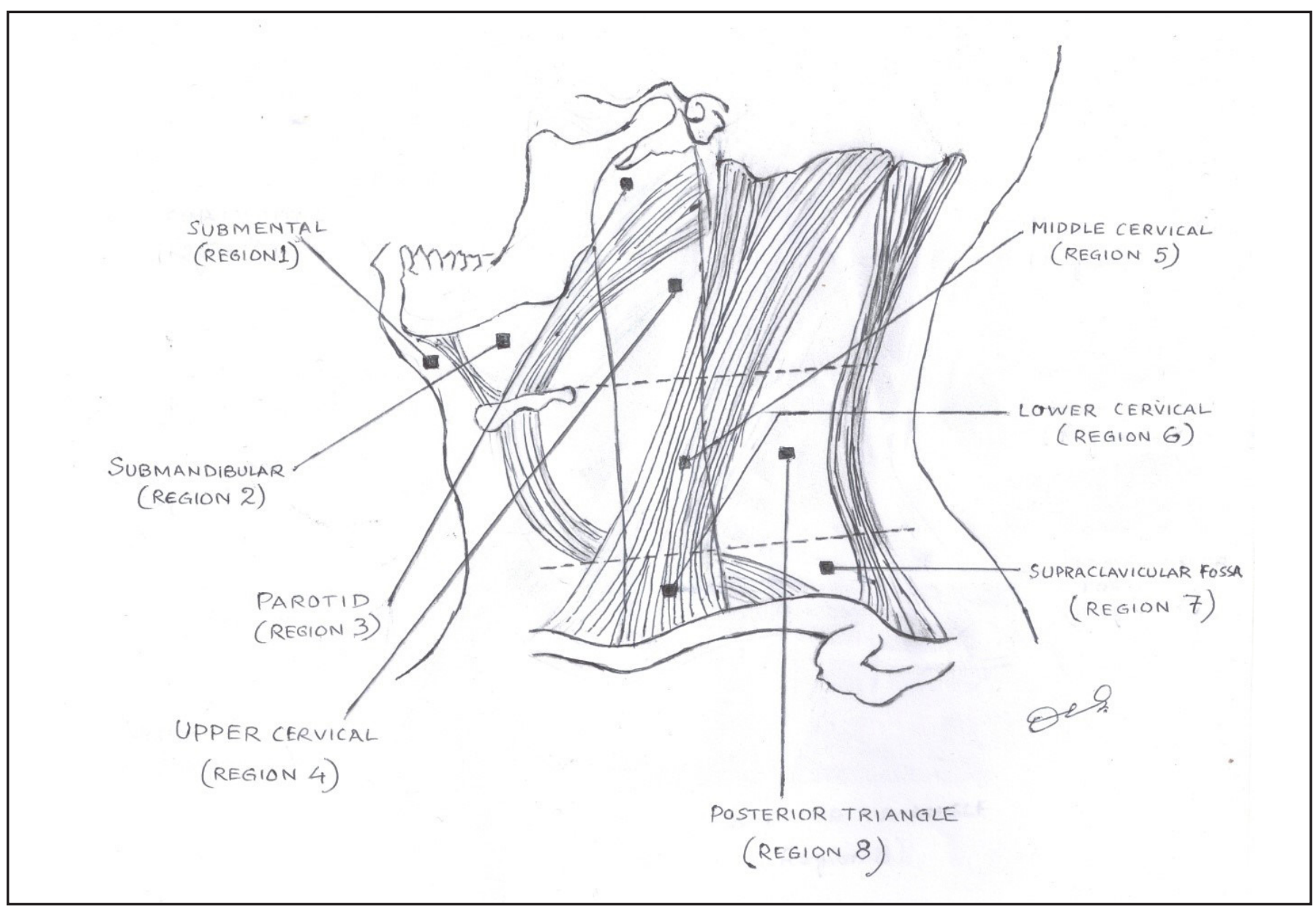

Fig.1. The sonographic zones of cervical lymph nodes (Image courtesy - Dr Debasish Guha)

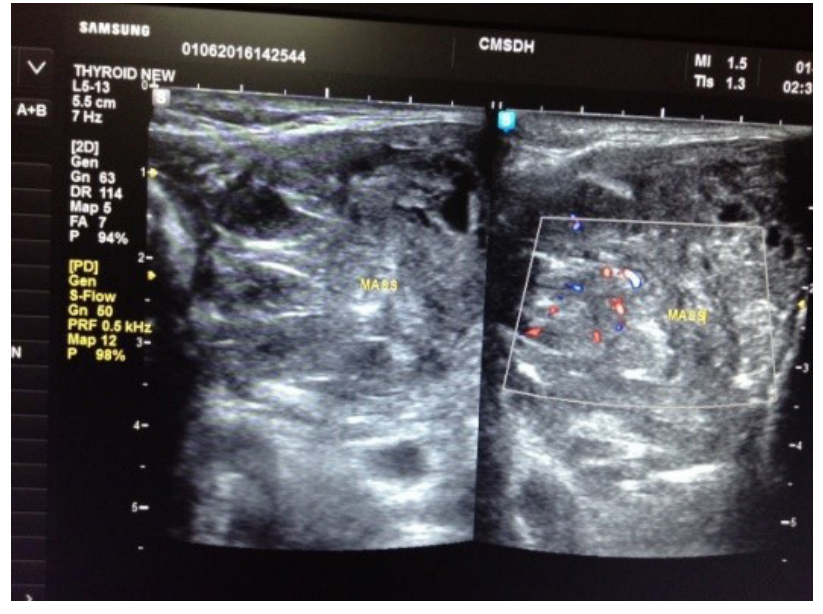

Fig.2. Grayscale and colour Doppler assessment of neck masses sensitivity and specificity of detecting neoplastic nodes are $83 \%$ and $98 \%$ respectively. Ariji et $\mathrm{al}^{5}$ advocated the following algorithm for classification of suspicious neck nodes for proper management. [Table I]

Since head and neck cancers and many nonneck cancers (such as stomach) metastasise to the cervical region metastasis and lymphoma are common differential diagnoses in patients presenting with neck nodes. Calcification in metastatic lymph node is not common, exception being thyroid malignancies. The papillary thyroid cancers show peripherally located fine punctate calcification which is sometimes accompanied with acoustic shadowing. Gooding 6 noted that previously resected medullary carcinoma may present with recurrent lymph nodes showing intranodal calcification. Post radiotherapy or chemotherapy nodes 


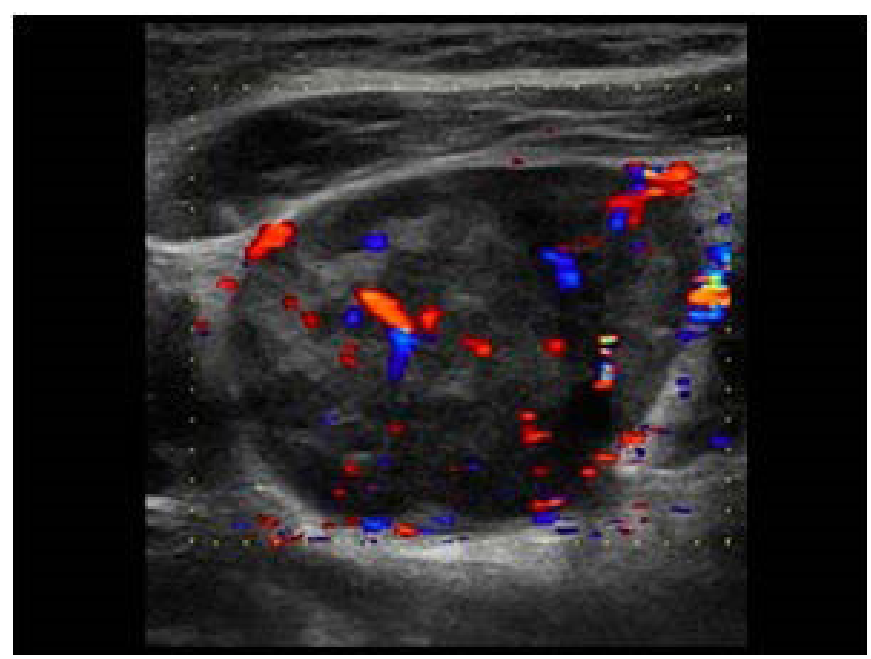

Fig.3. Colour doppler parenchymal pattern of flow

may show intranodal calcification.

Sometimes the malignant lymph nodes may show focal cortical hypertrophy or central necrosis. The lymph nodes are vascular but they show high pulsatility and resistive indices. Though malignant lymph nodes are hypoechoic metastasis from thyroid shows some variations. Metastasis from papillary carcinoma is usually hyperechoic and most commonly associated with microcalcification. The metastasis from solid papillary carcinoma may be cystic. Nodes show fine crystalline calcification in contrast to medullary where the calcification is coarse, which gives acoustic shadows. The malignant or metastatic lymph nodes are usually discrete but extra nodal spread may turn the margin fuzzy and there might be oedema surrounding the affected nodes. Metastatic lymph nodes are site specific and uncommon sites usually involves worse prognosis. Regardless of the primary presence of one lymph node metastasis in neck reduces the survival by $50 \%$ and another contralateral node by further $25 \%$.

Uncommon diseases Kikuchi, Kimurai and Rosai Dorfman disease may present with inflammatory lymphadenopathy. The key to diagnosis lies in a properly done FNAC, or needle biopsy.

Some centres may prefer ultrasound-guided FNAC for cytological diagnosis. Ultrasound-guided $\mathrm{FNAC}^{7}$ has been shown to be an accurate method in evaluation of cervical lymphadenopathy with a high sensitivity (89-98\%), specificity (95-98\%) and accuracy (95-97\%). This technique provides more accurate information than direct FNAC, and influences the therapeutic options. It has been reported that ultrasound-guided FNAC can correctly stage the cervical lymph nodes in $93 \%$ of the patients with head and neck carcinoma. In non- head and neck cancer, it has been found that ultrasoundguided FNAC has the risk of missing occult metastases in about $18 \%$ cases. Therefore, ultrasound-guided FNAC is useful in the follow-up examination of the neck after tumour excision, and serial examinations should be performed if no elective treatment of neck is undertaken.

\section{Assessment of the Thyroid Gland}

Thyroid nodules (Fig. 4) are very common. These are found in $4 \%-8 \%$ of adults by means of palpation, in $10 \%-41 \%$ by means of US, ${ }^{8}$ and in $50 \%$ by means of pathologic examination at autopsy. ${ }^{9}$ Most of these nodules are benign. USG of thyroid has fascinated the radiologist and surgeons for its popularity, usefulness and sensitivity (83\%). In spite of its high sensitivity US should not be utilised as a routine screening but palpable nodule may be recommended for US investigation, as US thyroid does not decrease the likelihood of cancer detection. The Society of Radiologists in Ultrasound consensus joint statement concluded that any lesion which is distinguishable from the surrounding is a nodule. ${ }^{10}$ They did not take into account the modalities for dealing with nodules less than $1 \mathrm{~cm}$ because of its slow growing nature and excellent prognosis.

The American Association of Clinical Endocrinologists (AACE) and Associazione Medici Endocrinologi (AME) recommendations of ultrasound of thyroid are as follows: ${ }^{11}$

1. To confirm presence of a thyroid nodule when physical examination is equivocal.

2. To characterize a thyroid nodule(s), i.e. to measure the dimensions accurately and to identify internal structure and vascularisation.

3. To differentiate between benign and malignant thyroid masses, based on their sonographic 


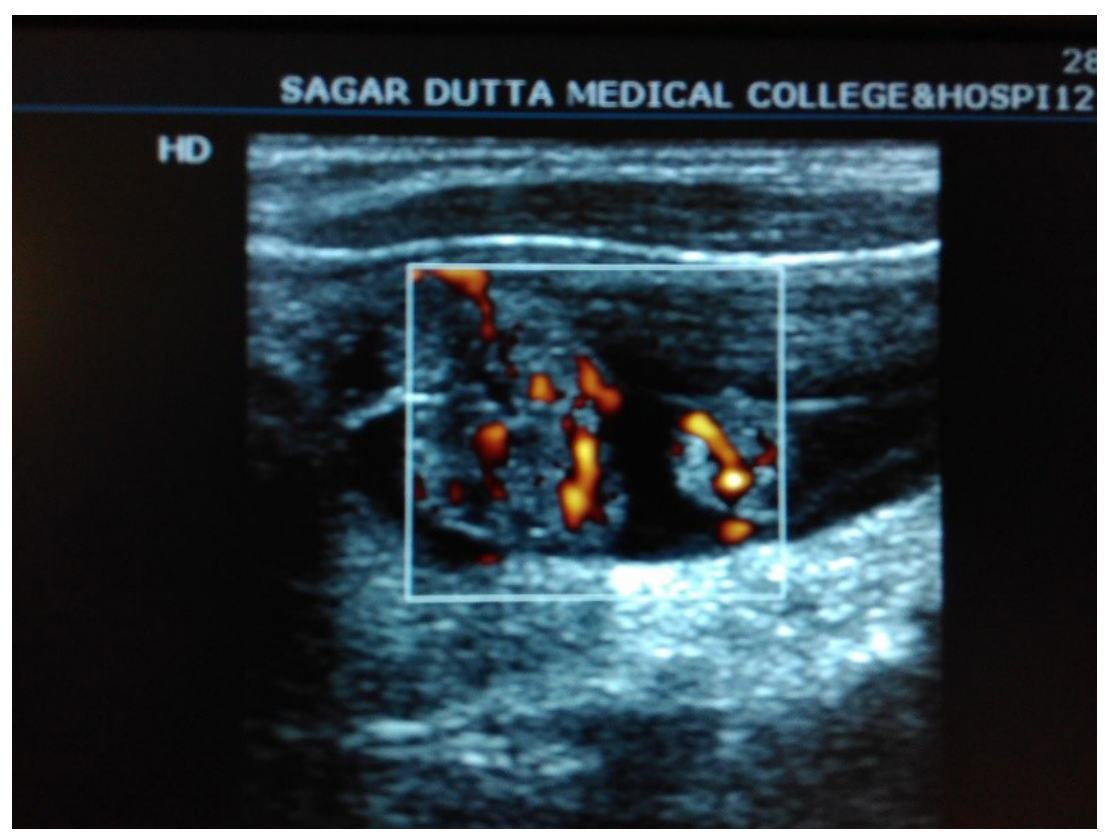

Fig.4. Thyroid nodules with intranodal vascularity

appearance.

4. To differentiate between thyroid nodules and other cervical masses like lymphadenopathy, thyroglossal cyst and cystic hygroma.

5. To evaluate diffuse changes in thyroid parenchyma.

6. To detect post-operative residual or recurrent tumor in thyroid bed or metastases to neck lymph nodes.

7. To screen high risk patients for thyroid malignancy like patients with history of familial thyroid cancer, multiple endocrine neoplasia (MEN) type II and irradiated

8. To guide FNABC or for therapeutic intervention.

Thyroid ultrasound cannot distinguish a hot or a cold nodule but it is a sensitive device for detection and determines benignity of thyroid masses. The benign nodule is usually either hypoechoic or echogenic, has a hypoechoic halo round it, shows curvilinear calcification and spoke of wheel vascularity. They are wider than longer and sometimes show comet tail artefacts. Malignant nodules lack the hypoechoic halo, are very hypoechoic with an irregular outline and show intrinsic vascularity. An avascular nodule is unlikely to be malignant. The nodules are longer than wider and show microcalcification. The other features suggestive of malignancy are their size $(>1 \mathrm{~cm})$ and local or lymph node invasion and flow in the central part of the tumour. Nodal metastasis is common in thyroid malignancy, usually the metastatic lymph nodes due to Ca thyroid involve levels II, III or IV (anterolateral groups). Microcalcification is highly predictive of malignancy and in USG they are seen as punctate hyperechogenicities and common in medullary or papillary carcinoma. Thyroid micro calcifications are psammoma bodies, which are $10-100 \mu \mathrm{m}$ round laminar crystalline calcific deposits .The calcification in the medullary carcinoma thyroid is usually coarse. Rarely follicular carcinoma may present with jugular vein thrombosis (Fig. 5). Multiplicity of nodules is not a boon and there is no guarantee that they are nonmalignant. ${ }^{12}$ Anaplastic thyroid carcinoma and lymphoma are highly aggressive. Thyroid lymphoma is usually paucivascular. Metastasis to thyroid is very uncommon. Independent predictors of the presence of malignancy in thyroid nodules in the Ultrasonography is done by studying 7 features- 1 ) irregular or microlobulated margin 2) microcalcification 3) hypoechogenicity 4) markedly low echogenicity 5) Larger than wider size 6) solidity 7) calcification.

Multinodular and diffuse goiters are common among 


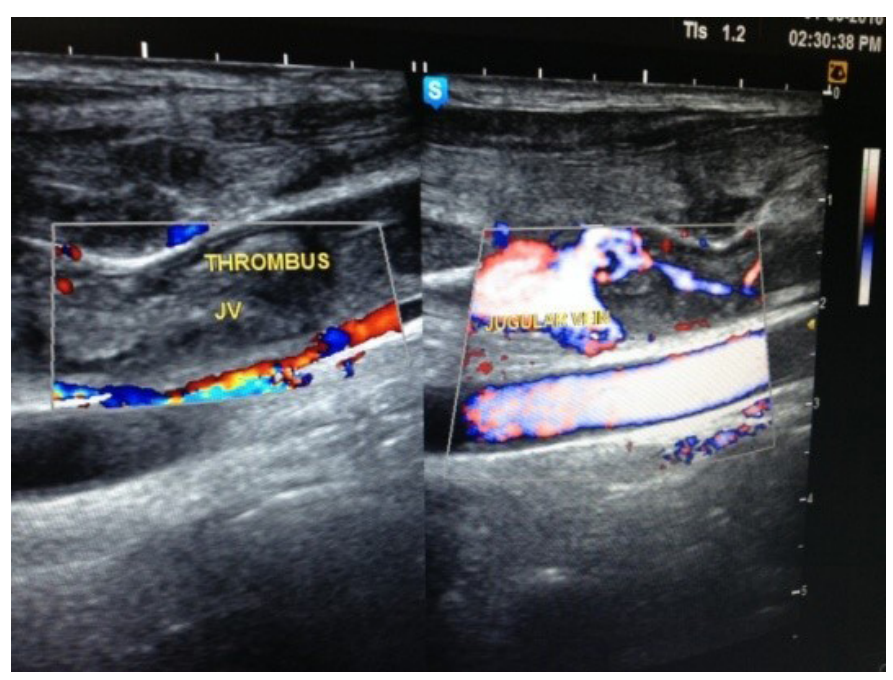

Fig.5. Thrombus in jugular vein in a patient with recurrent neck cancer

women of 35-50 years. USG predictability is high and sometimes no other invasive study is required for confirmation of their benignity. The thyroid gland is asymmetrically enlarged, with or without nodules. There are usually many nodules with barely any normal thyroid tissues. The nodules are prone to degeneration showing clear fluid inside; sometimes they accompany curvilinear calcification or comet tail artifact.

Graves' disease, chronic lymphocytic thyroiditis and de Quervain's thyroiditis present with goiter but their ultrasound features are not distinctive. In Graves' disease thyroid enlargement is massive, usually twice or thrice the normal size, and are highly vascular (thyroid inferno). The Hashimoto's disease presents with painless enlargement- they are vascular and may have numerous micronodules. The de Quervain's thyroiditis presents with a painful swelling and USG shows focal hypoechoic nodules, which are usually avascular. Thyroiditis is usually accompanied by perithyroidal lymph node enlargement- most commonly level VI nodes. Hashimoto's disease may turn into malignant thyroid nodule and concomitant hypoechoic nodules should be dealt with caution. FNAC plays an important role for suspicious lesions of thyroid.

Thyroid swelling or presence of thyroid nodules is so ubiquitous that many workers have tried to find uniform guidelines for management. The fate of thyroid nodules are traditionally decided on the basis of three internationally accepted recommendationsthe Kim criteria, American Association of Clinical Endocrinologists criteria and the Society of Radiologists in Ultrasound recommendations. According to Kim criteria, FNAC should be considered in a nodule if any one of the 7 suspicious criterions is present. ${ }^{13}$ This is independent of the nodule size.

According to the Society of Radiologists in Ultrasound FNAC should be considered in the following conditions:

1. If nodule size $\geq 1 \mathrm{~cm}+$ microcalcification

2. If nodule size if nodule size is $1.5 \mathrm{~cm}$ or above plus completely or almost entirely solid or presence of coarse calcification

3. If size is $2 \mathrm{~cm}$ or more and if mixed solid-cystic components are present or entirely cystic with mural nodule and has grown substantially (usually taken to be $>3 \mathrm{~mm}$ or more increase in diameter) in the last 6 months

4. If nodules of any size has concomitant abnormal lymph nodes (of any sizes).

The American Association of Endocrinologists guidelines show that FNAB should be performed on hypoechoic nodules with at least any one of the following additional US features - irregular margins, intranodal vascular spots, longer than wider shape or microcalcification.

Ahn et al has compared the three sets of guidelines and concluded that Kim and American Association of Clinical Endocrinologists criteria to be superior to the SRIU criteria. ${ }^{14}$ The Kim criteria are more sensitive and the American Association in Clinical Endocrinologists criteria more specific. ${ }^{14}$

Presence of cysts in thyroid should be evaluated cautiously and wherever required should accompany needle biopsy or FNABC. Indeterminate thyroid nodules can be evaluated by elastography. This technique utilises the intrinsic hardness of the nodule. Hard nodules are predictive of malignancy whereas a soft nodule is less likely to be malignant. In combination with colour Doppler and grey scale imaging, elastography can increase the sensitivity, specificity and positive predictive value, obviating the need for unnecessary FNABC. USG has some roles in guided treatment 
procedures, such as alcohol ablation of cystic nodules. High intensity focused ultrasound (HIFU) is a technique of targeted thermal ablation of nodules. US-guided HIFU ablation is an effective and safe noninvasive treatment method for benign solid thyroid nodules.

\section{Assessment of Salivary Glands ${ }^{15}$}

The major salivary glands are easily accessible by US probes and can help in narrowing down the differential diagnoses. Though CT is the ideal investigation for salivary inflammation and MR for tumours, US can sometimes give final diagnosis. The examiner must, however, be conversant with the complex anatomy of the region. The parotid gland has a deep lobe which remains hidden from the scanning probe but superficial gland can be easily diagnosed by identifying the retromandibular veins and external carotid artery. Further the facial artery and veins pass through the glands. The submandibular gland lies in the SM triangle bounded by the digastric muscles. The facial artery and vein passes through it. The glands show no distinctive echo pattern but are diffusely echogenic. The ducts of both the parotid or submandibular glands normally remain collapsed and difficult to identify. In calculus disease the ducts are dilated and the calculus itself gives strong acoustic shadows.

In most inflammatory lesions of acute onset the glands are enlarged, inhomogeneous and studded with numerous small hypoechoic areas and the vascularity is generally increased. In chronic inflammation the gland size may be reduced. When the inflammation is localised and there is gland necrosis, abscess might develop. Abscess is relatively easy to diagnose as the necrotic lesion is hypoechoic, may have an echogenic halo and shows posterior acoustic enhancement. The fluctuant mass sometimes show microbubbles within. Abscess can be easily drained by guided aspiration.

Chronic sialadenitis patients frequently present with recurrent pain and swelling. On US the gland is relatively smaller and shows in homogenous appearance consisting of hypoechoic lesions and increased blood flow. The differential diagnoses are Sjogren syndrome, disseminated lymphoma, metastasis and HIV associated infections. Tuberculous sialadenitis presents with a nonhmogeneous mass and necrotic caseous lesions will not show any color signal on Doppler US. Sialolithiasis is relatively easy to diagnose as the calculus in dilated duct can be identified readily by US. The sonography must clarify whether the calculus is in the parenchyma or duct to properly guide the treatment.

In all cases of Sialolithiasis there might be concomitant inflammation giving distinctive sonographic appearance. Other differential diagnoses are chronic sclerosing sialadenitis and actinomycosis. Intraparotid lymph nodes commonly pose diagnostic dilemma and unnecessary investigations follow, if careful examination is not done. In all doubtful cases, guided FNABC should be done.

The salivary gland tumours give nonspecific ultrasound appearance. Cysts are easily identified by its classic well defined hypoechoic clear appearance with posterior acoustic enhancement. Solid benign tumours show well defined heterogeneous mass. The most common benign salivary tumours occur in parotid and about 3/4th are pleomorphic adenoma. The tumours show a lobulated appearance and usually show no colour signal on Doppler study. There might be focal calcification within the mass. The other common tumours include Warthin tumour which show hypoechoic areas which are abundantly vascular. Pleomorphic tumours are usually lobulated, while Warthin tumours show anechoic areas. They present pictures which are distinctive but not pathognomonic.

Warthin tumours which appear as anechoic areas at US may occur in other benign tumors (pleomorphic adenoma), in malignant tumors (mucoepidermoid carcinoma), in an abscess or necrotic metastatic nodes. Warthin tumor may also appear in the form of a simple cyst at US and thus require differentiation from cystic carcinomas (mucoepidermoid carcinoma, acinic cell carcinoma) and benign cysts (lymphoepithelial cysts). The other differential diagnoses are lymphoma and metastasis which when present show nonspecific appearance and must be evaluated by guided FNAC. The common primaries metastasising in the salivary glands are head and neck cancers, breast, melanoma and rarely renal cell carcinoma. Salivary tumours are uncommon in submandibular gland but when present, most are 
malignant. Another problem frequently encountered in the US clinic is a recurrent painful parotid in chronic alcoholic, cirrhosis, endocrine disease or malnutrition. The condition, sialosis, presents as enlarged hyperechoic gland without colour Doppler abnormality where the deeper portion of the gland is poorly visualised.

There are many other benign neoplasms of the salivary glands which offer no specific US pattern. However, haemangioma demands special mention as it is sometimes in infants. The tumour is echogenic and extremely vascular. Phleboliths and tortuous blood vessels may be found in the tumour.

\section{Assessment of Larynx and Pharynx}

US examination has very limited role in the assessment of laryngeal and pharyngeal pathology. However, laryngeal masses, vocal cord thickening and paralysis can be assessed by US. Thyroid cartilage invasion and extra-nodal spread can be assessed by US. ${ }^{16}$ US has been successfully used for assessment nasopharyngeal carcinoma. ${ }^{17}$ It is seen to be highly sensitive but less specific and MRI is much better than US.

\section{Conclusion}

US is an important, sensitive and specific diagnostic aid for assessment of the neck masses. In experienced hand it is a very potent instrument providing diagnostic and prognostic leads for proper management of the patients. US can be successfully used for guiding FNABC and biopsies in suspected cases of neck masses.

\section{References}

1. Ahuja AT, Ying M. Sonographic Evaluation of Cervical Lymph Nodes. Am J Roent. 2005; 184(5): 1691-1699

2. Solbiati L, Rizzatto G, Bellotti E, Montali G, Cioffi V, Croce F. High-resolution sonography of cervical lymph nodes in head and neck cancer: criteria for differentiation of reactive versus malignant nodes. Radiology. 1988; 169(P):113

3. Vassallo P, Wernecke K, Roos N, Peters PE. Differentiation of benign from malignant superficial lymphadenopathy: the role of high-resolution US. Radiology 1992; 183:215-20
4. Yonetsu K, Sumi M, Izumi M, Ohki M, Eida S, Nakamura T. Contribution of doppler sonography blood flow information to the diagnosis of metastatic cervical nodes in patients with head and neck cancer: assessment in relation to anatomic levels of the neck. AJNR Am J Neuroradiol. 2001 Jan;22(1):163-9

5. Ariji Y, Kimura Y, Hayashi N, Onitsuka T, Yonetsu K, Hayashi K. et al. Power Doppler Sonography of Cervical Lymph Nodes in Patients with Head and Neck Cancer. AJNR Am J Neuroradiol 19:303-307 Gooding G A. Power Doppler Sonography of Cervical Lymph Nodes in Patients with Head and Neck Cancer : AJNR Am J Neuroradiol. 1998; 19:303-7

6. Gooding G A :Sonography of the thyroid and parathyroid, Radiology 1984; 151: 741-5

7. Knappe M, Louw M, Gregor RT. Ultrasonography-guided fineneedle aspiration for the assessment of cervical metastases. Arch Otolaryngol Head Neck Surg. 2000; 126(9):1091-6

8. Wiest PW, Hartshorne MF, Inskip PD, et al. Thyroid palpation versus high-resolution thyroid ultrasonography in the detection of nodules. J Ultrasound Med. 1998; 17:487-96

9. Horlocker TT, Hay ID. Prevalence of incidental nodular thyroid disease detected during high-resolution parathyroid sonography. In: Medeiros-Neto G, Gaitan E, eds. Frontiers in thyroidology. Vol 2. New York, NY: Plenum, 1985; 1309-12

10. Management of thyroid nodules detected at US: Society of Radiologists in Ultrasound consensus conference statement: Radiology 2005; 237:794-800

11. Available at: https://www.aace.com/files/thyroid-guidelines. pdf

12. Bonavita JA, Mayo J, Babb J, Bennett G, et al. Pattern recognition of benign nodules at ultrasound of the thyroid: which nodules can be left alone? Am J Roent. 2009; 193(1): 207-13

13. Kim EK, Park CS, Chung WY, Oh KK, et al. New sonographic criteria for recommending fine-needle aspiration biopsy of nonpalpable solid nodules of the thyroid. Am J Roent. 2002; 178(3):687-91

14. Ahn SS, Kim EK, Kang DR, Lim SK, et al. biopsy of thyroid nodules: comparison of three sets of guidelines. Am J Roent. 2010; 194(1): 31-7

15. Bialek EJ, Jakubowski W, Zajkowski P, Szopinski KT, Osmolski A. US of the major salivary glands: anatomy and spatial relationships, pathologic conditions, and pitfalls. RSNA education exhibit, May-June 2006

16. Hu Q, Zhu SY, Zhang Z, Luo F, Mao YP, Guan XH. Assessment of glottic squamous cell carcinoma: comparison of sonography and non-contrast-enhanced magnetic resonance imaging. Journal of Ultrasound in Medicine 2011; 30:1467-74

17. Pan D, Zhu SY, Xu YB, Wu YF, Lun HM, Wei YY. Sonographic findings of nasopharyngeal carcinoma and its involvement in the parapharyngeal space. Journal of Ultrasound in Medicine 2013; 32:1041-47; doi:10.7863/ultra 